\title{
The Teaching Strategies for Metaphorical Competence of Chinese College English Learners in Internet+ Era
}

\author{
Qing Wei \\ Zhejiang Yuexiu University, Shaoxing, China \\ Email:20131050@zyufl.edu.cn
}

How to cite this paper: Wei, Q. (2021) The Teaching Strategies for Metaphorical Competence of Chinese College English Learners in Internet+ Era. Open Access Library Journal, 8: e7816.

https://doi.org/10.4236/oalib.1107816

Received: August 1, 2021

Accepted: September 3, 2021

Published: September 6, 2021

Copyright $\odot 2021$ by author(s) and Open Access Library Inc.

This work is licensed under the Creative Commons Attribution International License (CC BY 4.0).

http://creativecommons.org/licenses/by/4.0/

\section{(c) (i) Open Access}

\begin{abstract}
Internet+ new era brings both opportunities and new requirements for College English Teaching in China. According to the latest college English Teaching Guide (2020 Edition), much importance should be attached to cultivating students' English application ability and enhancing their cross-cultural communication ability. In order to achieve this goal, the cultivation of foreign language metaphor ability is indispensable. Based on the conceptual metaphor theory and the definition of metaphorical competence in the field of cognitive linguistics, and the current level of College Students' English Metaphorical Competence in China, this paper puts forward the top-level design concept and the explicit and implicit metaphorical competence teaching strategies, so as to improve the level of college students' foreign language metaphorical competence in China.
\end{abstract}

\section{Subject Areas}

Language Education

\section{Keywords}

College English, Metaphorical Competence, Teaching Strategies

\section{1. “互联网+”时代下的大学英语教学}

2015 年, 第 1 届国际教育信息大会和第 15 届中国教育信息化创新与发展 论坛分别于 5 月份和 10 月份召开, 两次会议均指出信息化技术和互联网时代 为教育注入了新的活力, 带来了革命性的影响。互联网、大数据、云计算等 信息技术的发展与普及不仅使人们工作、日常生活、交际方式等发生了巨大 
的变化, 更是对传统的教与学产生了前所未有的影响。进入了互联网+新时代, 传统的单向知识传递方式收到了质疑和挑战, 双向或多维的知识习得模式逐 渐取而代之(教师不再是知识唯一传授者, 互联网、同伴等都可以是知识的来 源)。英语课堂教学日趋多元化, 教学资源越来越丰富, 个性化学习和因材施 教等教学教育理念不断改革和创新, 这些为我国外语教学课堂教学效果和学 生英语能力的全面提升提供了新的路径和方向。

互联网+新时代在为教育注入新鲜血液和创新动力的同时, 也对教育教学 目标提出了新的发展要求。大学英语教学指南 2020 版中对大学英语教学新时 代下的新教育使命和目标给与了清晰的描述。指南中指出总体目标之一是培 养学生的英语应用能力, 增强跨文化交际意识和交际能力。就大学外语教学 而言, 切实可行的做法是将跨文化交际能力培养融于日常的语言教学的各项 活动与环节当中。其中, 最有效的途径之一就是隐喻教学 [1]。

\section{2. 隐喻及隐喻能力}

\section{1. 隐喻理论发展动态}

隐喻的研究由来已久, 最早可追溯到公元前 400 年。但 Lakoff and Johnson (1978)提出的 “概念隐喻理论” (Conceptual Metaphor Theory/CMT), 开启了 隐喻的认知研究视角。CMT 认为隐喻是储存在人们头脑中的一种经过归纳 的、反映事物本质以及不同概念域之间映射关系的隐喻性表达，它们构成了 人们日常思维和行动的基础，是我们赖以生存的方式。例如，Life is a journey 是概念隐喻而 “I just took an unexpected direction, my life is going nowhere, she'll go places in life, she's gone through a lot in life” 这句话则是其在语言层面 的体现。概念隐喻受认知语言学中的生成学派 “规则制约表达” 影响很大, 其研究的任务之一便是找出自然语言中的隐喻性话语基本规则, 因此 CMT 研究侧重隐喻的语言和其概念维度, 忽略了隐喻的交际层面。

当越来越多的研究者关注 CMT 的不足和其自身存在的悖论时, 注重隐 喻的语言、认知和交际三维度的刻意隐喻理论(Deliberate Theory/DT) Goatly (1997)、Shen \& Balaban (1999)、Cameron (2003)、Charteris Black \& Musolff (2003)和 Goddard (2004)便应运而生[2]。刻意隐喻理论以隐喻使用所激活的 元语言属性为出发点, 将交际维度吸纳于传统的隐喻认知范畴之中, 建构了

“语言、概念与交际” 三维支撑的全新隐喻分析平台, 旨在提取隐喻认知背 后的精细化机制, 实现隐喻认知研究的分科思辨与科学验证, 并在跨学科背 景下促进隐喻研究走向多元融合 [3]。

近些年来随着基于使用语言观的不断发展以及语料库语言学的发展, 一 些学者受其启发, 开始将基于使用的研究方法和范式应用到概念隐喻中来, 试图阐释概念隐喻理论所不能回答的问题。美国新墨西哥大学语言学专家 Daniel Sanford 教授(2008)在基于使用语言观和范式理论的基础上，提出涌现 隐喻理论(Emergent Metaphor Theory/EMT)。EMT 认为隐喻是连接认知语域 (cognitive domains)的图式, 隐喻频率的差异是隐喻系统的核心方面, 隐喻在 整体映射(overall mappings)和个体话语(individual utterances)层面上均受频率 
效应(frequency effects)的影响。[4] EMT 将研究聚焦在语言的实际使用而非潜 在的、已经预设好的先验结构中。这就为概念隐喻理论中的出现的难以理解 的隐喻结构找到了解释。换而言之, 基于使用语言观的 EMT 认为隐喻是受频 次驱动的一种浮现结构, 即是在语言的使用中涌现出来的, 也就是说隐喻的 习得是思维和语言两个层面同时并相互作用下发生的。

认知语言学下的概念隐喻理论不断被完善和发展, 无论是 DT 还是 EMT, 都是对 CMT 的延伸, 从更多的视角解读隐喻。这也为二语和外语教学领域 内的隐喻研究提供了更多的理论支撑。

\section{2. 隐喻能力}

认知隐喻研究者们发现日常会话中每分钟就会有 4 个隐喻出现(Pollio, 1977), 且不同语言隐喻的使用方式亦相差较大, 因此恰当地理解和使用隐喻 便成为语言学习中的一项必备能力。隐喻能力被认为是继 Chomsky 的语言能 力(linguistic competence)、Hymes 的交际能力(communicative competence)之 后语言学习者的第三大能力, 已发展成为国际应用认知语言学界的焦点话题。 Danesi (1986)认为隐喻能力是指识别和使用新型隐喻的能力, 比如在恰当的 语境中使用隐喻以及使用策略等。王寅和李宏(2004)认为隐喻能力不仅是指理 解和学习隐喻的能力, 还包括丰富想象力和创新思考能力。袁风识(2012)从隐 喻能力的本质特征出发, 将其解释为认知主体基于自身体验在两个不同类型 的认知对象之间建构起语义关联的一种能力。[5] Littlemore 和 Low 对何为“隐 喻能力”给出了更具体更详尽的具有可操作性的解释(Littlemore \& Low 2006)。 他们认为隐喻能力主要包含以下 4 个方面: (1) 使用隐喻的创造性, 即创新隐 喻的能力; (2) 理解隐喻的熟练度, 指理解一个隐喻多层含义的能力; (3) 理 解新隐喻的能力, 指正确理解 “原创性” 隐喻的能力; (4) 理解隐喻的速度, 指轻松、准确、及时理解隐喻的能力 [6]。

综上所述, 隐喻能力能够反映一个人的交际和语言能力, 因此隐喻能力 对语言学习来说至关重要。以英国伯明翰大学 Jeannette Littlemore 博士为代 表的学者对一语和二语语言使用者的隐喻能力进行了大量的实证研究, 并取 得了丰硕成果：1) Littlemore (2001)发现，非本族语者往往根据图示和语境信 息对隐喻进行理解, 尤其在碰到模棱两可的意义时, 语言、文化背景的差异 会造成理解的偏误；2) Littlemore (2004)注意到, 在英国大学课堂国际学生经 常难以理解有关课堂内容和教师态度的隐喻用法; 3) Littlemore (2010)的研究 表明, 本族语者产出规约型隐喻的几率远远高于非本族语者；4）Littlemore, Chen, Koester \& Barnden (2011)探讨了大学课堂讲演中的隐喻使用及其对非 本族语者理解造成的困难。[7]因此母语使用者与非母语使用者在隐喻能力上 有显著差异性。Hashemian \& Nezhad (2007)强调, 应加强培养二语学习者对 二语概念系统与一语概念系统的体验相似性和文化差异性的意识。隐喻意识 及隐喻理解与产出能力对二语学习者跨文化交际能力具有不可估量的影响。 研究者们开始重点关注隐喻意识的培养以及隐喻能力的提高, 从而提高外语 教学的效率 [8]。 


\section{3. 隐喻能力的可教与可学性}

隐喻是否具有可教性至今在研究界中未达成一致意见。亚里士多德认为 隐喻是无法教授给他人的。Hawkes (1984)认为隐喻的使用是一种捕捉两个概 念领域中的相似性的能力, 这种能力取决于一个人的认知, 因此无法通过教 授习得。蒋楠(2004)认为成年二语学习者的母语概念语义会自动干扰甚至阻碍 二语概念语义系统的发展, 因此他们很难摆脱对母语的以来, 这样二语学习 中隐喻的使用只会加强二语与母语的结合, 并使其逐渐固化。更不用说在外 语环境下的语言学习者。他们接触和使用外语的机会少之又少, 因此二语和 外语学习者要想通过课堂学习获得如母语使用者一样的隐喻能力是极其困难 的[9]。

然而更多的学者和研究者认为隐喻能力是可以培养的。Mahon (1999)驳 斥了亚里士多德观点, 提出每个人都可以创造并使用隐喻。Kovecses and Szabo (2002)在研究中发现在语言教学中, 只要给予隐喻足够的关注, 隐喻能力便可 获得提升。Pavlenko (2009) 认为对于母语与二语中概念不对等的语义结构, 只 要有充足的二语学习和输入, 学习者能将两种语言中相联系的概念进行重组, 从而形成新的概念, 达到概念流利。吴丽丹(2007)指出外语语言学习本应就是 隐喻能力发展的过程, 因为语言形式的学习会促使学习者通过语言感知、体 验新的隐喻思维方式, 从而形成相应隐喻思维习惯。[10] Littlemore et al. (2012, 2014)对不同外语水平学习者隐喻能力的研究也证实了二语学习者的隐 喻能力是可以学的。语言隐喻教学可构筑学习者二语概念, 有效提高学习者 对概念隐喻及其相应的概念系统的了解和掌握, 提高其二语隐喻能力。

基于使用语言观的涌现隐喻理论指出, 隐喻同其他语言结构一样, 是在 使用中涌现出来的。这为隐喻的可教与可学提供了新的理论支撑, 也为外语 教学中隐喻能力培养和教学提供了新的启示。

\section{4. 中国大学生英语隐喻能力现状}

隐喻能力是交际能力的重要体现, 是二语和外语学习者自然得体流畅使 用目标与的表现, 也是语言理解和表达能力的更高要求。因此近些年来, 我 国语言学研究者们在理论和实践层面上不断探讨隐喻能力与外语教学的关 系, 如何培养隐喻能力以及培养隐喻能力的重要性。虽然我国外语教学水平 不断提升, 但大学生英语隐喻能力水平整体依然偏低。丁川(2007)研究发现中 国英语学习者的隐喻能力与英语母语使用者相比存在显著差异。[11]吕燕 (2008)研究结果发现高语言水平和低语言水平组的外语学习者在隐喻理解和 隐喻产出上均表现能力不足。[12]王鹏(2011)的研究也同样发现参与者的隐喻 创造能力偏低。[13]刘晶媛(2014)的实证研究发现受试者的隐喻识别能力高于 隐喻解释和产出能力, 但总体隐喻能力依然偏低。[14]因此, 如何开展隐喻教 学并有效提升英语隐喻能力依然是我国大学英语教学的亟需解决的课题。陈 朗(2016)基于文献统计, 剖析了国内隐喻能力研究之现状, 强调研究的必要性、 迫切性。[6]杨娜(2018)提出学习型词典介入式外语隐喻能力自主发展的路径 与方法, 该研究不仅对推进外语隐喻能力研究的体系化与规模化发展有所禆 
益, 而且有助于词典介入式外语隐喻能力自主发展模式的创新构建。[15]李文、 郭建辉(2020)对中国高级英语学习者概念隐喻能力(基于中外博士论文的研 究)中指出, 虽然国内高级英语学习者总体具备较强概念语法隐喻能力, 但依 然存在名词化使用过多、部分类型使用不足、语法隐喻词汇多样性较低以及 隐喻转化错误或不完整问题[16]。

\section{3. 大学英语课程中隐喻能力培养}

\section{1. 顶层设计理念 ${ }^{1}$}

隐喻能力是外语学习者具备优异的语言能力和跨文化思辨能力的保障。 然而现有的外语教材, 外语教学大纲, 外语测试和评价等均未系统地体现隐 喻知识教授和隐喻能力培养, 因此导致隐喻能力的重要性在思想上和实践上 多是纸上谈兵, 鲜有落地生根。虽然许多学者和一线教师在课堂教学中进行 了隐喻教学, 但多是单一的, 短期的, 非系统性的, 最终效果甚微。所以我 国大学英语学习者隐喻能力的整体提升必须要 “顶层设计”。例如：1）大学 英语教学大纲和目标中要明确隐喻教学, 给出具有指导性和可操作性的隐喻 能力培养方案。最新大学英语教学指南中明确指出各高校可依据指南, 结合 学校、院系和学生的实际情况, 确定具有本校特色的教学目标和教学要求。 因此各高校的大学英语教学中可依据自身定位及人才培养要求, 学生现有英 语水平, 制定出切实可行的个性化的隐喻教学大纲和目标。2) 未来大学英语 教材应将隐喻能力这一要素纳入编写原则中, 系统性地体现隐喻知识, 增加 利于培养学生隐喻能力的语篇和有针对性地练习任务。3) 大学英语课程评价 要检测学生隐喻能力水平。评价是隐喻教学目标是否落地, 隐喻能力是否提 升的有效反馈。课程评价结果又将为隐喻教学目标的完善和隐喻能力培养方 案的优化提供数据支撑。因此课程测试和考核中应突出对学生隐喻能力的测 试。

此外, 顶层设计理念的原则是自上而下, 在隐喻教学中, 教师作为主体, 发挥着主导和引领作用, 因此教师自身的隐喻意识和隐喻素质至关重要。大 学英语教师除了要加强自身隐喻意识, 更要提升自身的隐喻素质, 具备系统 的隐喻知识, 知晓最新隐喻相关研究成果, 并能将隐喻教学目标有效地落实 到语言教学中。这既需要教师自身具有好学品质，同时也需要专业指导与训 练。

\section{2. 坚持显性与隐性隐喻教学相结合}

Achard M (2014)指出, 应用认知语言学理论带来两种不同的语法(言)教 学观：一种是语法就是概念化、语言习得就是概念习得理论指导下的自上而 下的演绎式显性教学; 另一种是基于使用的语言习得观指导下的自下而上的

\footnotetext{
${ }^{1}$ 百度百科对顶层设计的解释为: 运用系统论的方法, 从全局的角度，对某项任务或者某个项目 的各方面、各层次、各要素统筹规划，以集中有效资源，高效快捷地实现目标。束定芳教授在 《中国外语教学改革与发展: 顶层设计与无形之手》中指出，“顶层设计可以指不同层面、不同 方面的规划与决策。顶层设计可以包括政策方针, 也可以是具体的实施方案。就外语教学而言, 各个高校、各个区域，甚至各个专业都可以并应该有顶层设计” [17]。
} 
归纳式隐性教学。[18]外语教学中隐喻能力习得与母语隐喻能力习得的相似与 差异性决定了大学英语课程中隐喻教学要兼具这两种方式。

外语隐喻能力的发展有赖于隐喻信息的有效呈现。显性隐喻教学方式主 要包括: 1) 教师系统性地讲解隐喻知识, 向学生普及隐喻在语言学中的重要 性, 同时教师也要关注最新的隐喻研究成果, 更新隐喻知识, 提升学生的隐 喻意识。2) 以教材为蓝本, 教师从词汇, 句子以及语篇等三个方面向学生讲 解课文中所涉及的隐喻, 并通过针对性的练习巩固和提升学生的隐喻能力。 外语隐喻能力与外语词汇认知系统构建乃至外语词汇深度习得有着密切的关 系。因此词汇层面的隐喻教学是学生外语隐喻能力有效提升的第一步也是重 要的一步。在进行词汇隐喻教学时, 应优先选择语义被隐喻性使用的词汇, 让学生在语境中了解词语的本义以及引申义(即隐语性表达)用法。例如 Corruption corrodes public confidence in a political system。corrode 一词在这 句话中就呈现了它的隐喻性含义, 即削弱。但它本义是腐蚀, 因此, 这样学 生就掌握了该词汇自身内部层面的隐喻信息。随后教师应以同样方式, 设置 一些练习, 给出词汇的本义, 然后让学生猜测其在句子中的意思。在练习中, 必然会涉及到中西思维中映射的差异, 这样就从词汇层面上升到了概念体系 层面, 这种显性的教学方式就有效地减少母语负迁移的干扰, 提升了学生的 外语隐喻整合能力。隐喻不仅体现在具体的词汇和语句中, 还体现在语篇中, 即文章的主题是否被隐喻。引导学生通过对单词, 短语以及句子等中隐喻性 的表达的整理与归纳, 上升到主题隐喻, 即概念思维层面, 进而习得中西两 种文化的异同, 培养学生隐喻能力的同时, 也推动了学生跨文化思辨能力的 发展。3) 配套的练习和考核方式必不可少。评估是教学环节中必不可少的一 环, 但很多教学设计中却忽视了这一环节的重要性。它是对教师教学效果和 学生学习效果的考核, 有形无形地指挥着课堂教学的内容和方式。隐喻教学 练习的设计原则坚持模仿与创新并行。根据教师教授的隐喻知识以及教材中 所涉及的隐喻运用实例, 模仿式的练习题则主要是检测学生对所讲授的知识 的识记和理解如何。该类练习题多以填空, 选择或简答题为主。创新式练习 题则侧重考察学生的隐喻应用能力, 隐喻性思维能力和跨文化交际能力, 以 话题式的作文或情境题为主。

最新的涌现隐喻理论/EMT 认为隐喻是在所使用的语言实例中涌现的, 说话者先前对隐喻的接触频次为后来隐喻性话语的处理起到了重要作用。也 就是说, 隐喻的产出与解释是通过频率效应来运作的, [4]因此在隐喻教学中, 隐性教学方式主要是通过大量输入具有隐喻含义的词汇, 短语, 以及含有大 量隐喻表达的文章给语言学习者。例如教师根据所教授单元的文章主题及所 输入的隐喻知识类型, 为学生提供相似的语篇阅读, 鼓励学生通过上下文语 境猜测熟词生义的方式, 归纳隐喻性表达语句等自我发现语言表面背后的中 西思维的异同, 最终实现潜移默化提升学生隐喻能力。

互联网+的时代下, 教育载体呈现了电子化, 网络化, 动态化等多模态化 的状态。因此, 大学英语教学应紧跟时代发展, 积极探索多模态化下的隐喻 能力培养方式的时尚化。隐喻不仅存在语言文本中, 还存在图画, 音频, 视 频等各种载体中。这大大丰富和增加了外语学习者学习资源以及接触和使用 
英语的方式与机会, 使得学习者对隐喻实例的接触和使用频率得以提高, 不 断固化头脑的隐喻认知图式。与此同时, 外语学习者也不断接触母语使用者 的新奇隐喻, 随着新奇隐喻被接触的次数不断增多, 他们头脑中的隐喻图式 的解释力就不断增强, 在实际交际中, 就避免了因隐喻能力不足而造成的跨 文化交际能力的缺失, 进而导致交际低效, 甚至产生误会或误用。

\section{4. 结语}

新的隐喻理论就像隐喻教学上的灯塔, 而互联网+时代的到来则为隐喻教 学提供了新的助力, 这一切都将使隐喻教学更加科学有效, 使我国大学生英 语隐喻能力整体水平不断提升。因此本文以隐喻以及隐喻能力研究的新动态 为指导, 借鉴其他学者有关外语教学中隐喻能力提升与培养的相关研究, 对 当前互联网+时代下的大学英语隐喻教学提出了如下两点思考:

一是借鉴顶层设计理念, 大学英语隐喻教学应坚持从全局的角度, 从教 学指南、教材编写、课程考核、教师培训等方面被统筹规划; 二是坚持隐性 与显性教学相结合策略。基于使用的语言观下的隐喻涌现理论认为隐喻就像 其他语言结构一样, 是在语言实例使用中涌现出来的。因而外语隐喻能力的 培养既需要教学中教师从概念、词汇、语法以及语篇层面进行显性有意识地 进行培养, 也需要频次发挥驱动力作用, 即隐形策略, 多渠道增加输入量, 实现潜移默化。此外, 互联网+时代下的教育的多模态, 为大学英语隐喻教学 效率的提升, 提供了有效途径。

\section{基金项目}

本文系浙江越秀外国语学院 2020 年度校级课题 “基于使用语言观视角下 的频次与大学生英语隐喻习得实证研究” (N2020036)的结题成果; 2021 年度 校级教育教改课题 “以 ‘关键能力’ 培养为目标的大学英语教学行动研究一 一基于课程思政背景” (XJJG2103)的成果。

\section{Conflicts of Interest}

The author declares no conflicts of interest.

\section{References}

[1] 刘正光. 大学外语教学跨文化交际能力培养的途径与隐喻教学 [J]. 东北师大学报 (哲学社会科学版), 2016(3): 121-124.

[2] 范振强. 基于交际维度的刻意性隐喻理论: 问题与展望[J]. 外语教学理论与实践, 2021(2): 1-10.

[3] 张建丽. “刻意隐喻理论”: 隐喻研究的最新进展. 2017. http://ex.cssn.cn/zx/201708/t20170829_3623339.shtml

[4] 孙毅. 涌现隐喻理论学术路向: 基于使用的认知研究新范式[J]. 外语研究, 2021, 38(1): 15-23.

[5] 陈朗. 基于英语演讲可比语料库的中国中高水平 EFL 学习者隐喻话语能力研究 [D]: [博士学位论文]. 上海: 上海师范大学, 2017.

[6] Littlemore, J. and Low, G. (2006) Metaphoric Competence and Communicative 
Language Ability. Applied Linguistics, 27, 268-294.

https://doi.org/10.1093/applin/aml004

[7] 陈朗. 国外隐喻能力研究综述一一回眸与前瞻 [J]. 外语研究, 2016, 33(4): 22-26+52.

[8] 任风雷. 隐喻意识, 隐喻能力和二语习得 [J]. 安徽广播电视大学学报, 2005(4): 98-100.

[9] 石进芳. 二语隐喻能力及其可学性和可教性: 回顾、分析与启示[J]. 外语教学理 论与实践, 2018(2): 44-50.

[10] 吴丽丹. 外语隐喻能力培养新探[J]. 广州大学学报(社会科学版), 2007, 6(9): 65-69.

[11] 丁川. 基于认知的中国英语学习者隐喻能力发展研究[D]: [博士学位论文]. 上海: 上海交通大学外国语学院, 2007.

[12] 吕燕. 英语作为外语语言学习者英语能力发展一一个案研究 [D]: [硕士学位论 文]. 上海: 东华大学外语学院, 2008 .

[13] 王鹏. 大学生英语能力及其触发英语的认知研究 [D]: [硕士学位论文]. 秦皇岛: 燕山大学外语学院, 2011 .

[14] 刘晶媛. 非英语专业大学生隐喻能力发展现状的实证研究[J]. 长春理工大学学报 (社会科学版), 2014(4): 179-180+183.

[15] 杨娜. 外语隐喻能力自主发展与学习型词典介入: 路径与方法[J]. 外语研究, 2018, 35(5): 23-27.

[16] 李文, 郭建辉. 中国高级英语学习者概念语法隐喻能力一一基于中外博士论文的 研究[J]. 外语教学理论与实践, 2020(1): 50-58.

[17] 束定芳. 中国外语教学改革与发展: 顶层设计与无形之手[J]. 山东外语教学, 2016, 37(2): 28-32.

[18] 石进芳. 显性/隐性隐喻输入对隐喻能力发展的比较研究[J]. 南昌航空大学学报 (社会科学版), 2015, 17(3): 91-98+124.

\section{Appendix (Abstract and Keywords in Chinese) 互联网+时代下中国大学英语生隐喻能力教学策略研究}

摘要: 互联网+新时代为我国大学英语教学既带来了机遇也提出了新的要求。 最新大学英语教学指南(2020 版)提出要注重培养学生的英语应用能力, 增强 跨文化交际沟通能力。为实现这一目标, 外语隐喻能力的培养必不可少。本 文基于认知语言学领域内的概念隐喻理论和隐喻能力定义, 及我国大学生英 语隐喻能力现状, 提出了顶层设计理念, 显性与隐性相结合的隐喻能力教学 策略, 以其提升我国大学生外语隐喻能力水平。

关键词: 大学英语, 隐喻能力, 教学策略 